\title{
Fledermausaktivität in Gondelhöhe in Bergwaldgebieten der Steiermark, Österreich
}

\section{Acoustic activity of bats at nacelle height in wind parks of Styrian montane forests, Austria}

\section{Senta Huemer und Brigitte Komposch}

\section{Zusammenfassung}

Die österreichische Klima- und Energiestrategie sieht einen massiven Ausbau der Windkraftproduktion vor. Die geplanten Standorte für den Ausbau in der Steiermark befinden sich hauptsächlich in Bergwaldgebieten. Bisherige Studien zum Thema Fledermausaktivität und Windkraft wurden vor allem im Tiefland oder in Mittelgebirgslagen durchgeführt. Aktuelle Untersuchungen aus dem Alpenraum zeigten teils beträchtliche Fledermausaktivitäten. Aus den Bergwäldern Österreichs liegen bis jetzt jedoch noch keine publizierten Daten zur Fledermausaktivität in Gondelhöhe vor. Ziel des vorliegenden Kapitels ist es, den Wissensstand darüber zu verbessern.

An fünf verschiedenen Standorten in Bergwäldern der Steiermark, Österreich, wurde die Fledermausaktivität in 1200 bis $1700 \mathrm{~m}$ über NN in Gondelhöhe mittels Batcordern (ecoObs) gemessen. Zusätzlich zur Fledermausaktivität wurden an vier Standorten die Windgeschwindigkeit und an zwei Standorten die Temperatur in Gondelhöhe erhoben. Die Standortauswahl erfolgte im Rahmen von Planungen für Windkraftprojekte. Der Erfassungszeitraum lag an allen Standorten zumindest zwischen Mai und Mitte September. An allen Standorten betrug der Rotor-Boden-Abstand maximal $50 \mathrm{~m}$.

\footnotetext{
S. Huemer $(\bowtie) \cdot$ B. Komposch

Institut für Tierökologie und Naturraumplanung OG, ÖKOTEAM, Graz, Deutschland

E-Mail: huemer@oekoteam.at

B. Komposch

E-Mail: komposch@oekoteam.at
} 
An den fünf Windparkstandorten wurden insgesamt mindestens acht Fledermausarten nachgewiesen. Das Artenspektrum umfasste sowohl ortstreue Arten wie Zwergfledermaus und Nordfledermaus als auch typische Langstreckenzieher wie Abendsegler, Zweifarbfledermaus und Rauhautfledermaus. An einem Standort wurde die Gattung Myotis aufgezeichnet. Es stammten 85,2-97,4\% der Aufnahmen von Vertretern der Gruppe Nyctaloid und 2,5-14,7 \% von Vertretern der Gruppe Pipistrelloid.

Es wurde eine hohe Variabilität der Aufnahmenzahlen verzeichnet, sowohl zwischen den Standorten als auch am selben Standort zwischen den Jahren sowie innerhalb eines Windparks. Die dokumentierte Fledermausaktivität schwankte nicht nur stark zwischen den einzelnen Monaten, sondern auch an einzelnen Tagen. Die höchsten Aktivitäten wurden in den Monaten Juni bis September gemessen. Im April und Mai wurden generell geringe Aktivitäten dokumentiert. Im Oktober wurden noch an zwei Standorten höhere Aktivitäten registriert. Die hohen Variabilitäten unterstreichen die Wichtigkeit mehrjähriger Erhebungen mittels Gondelmonitoring sowie mehrerer Gondelmonitoring-Standorte innerhalb eines Windparks.

Die höchsten Windgeschwindigkeiten, bei denen Fledermäuse noch aktiv waren, reichten von 6,5-15 m/s. Erwartungsgemäß nahm die Aktivität mit zunehmender Windgeschwindigkeit ab. Dieser Rückgang variierte jedoch stark standortabhängig und auch innerhalb eines Standortes von Jahr zu Jahr. Fledermausaktivität begann teilweise bereits um den Gefrierpunkt.

Diese Ergebnisse zeigen, dass Fledermäuse in Bergwäldern bei höheren Windgeschwindigkeiten sowie kälteren Temperaturen als in tieferen Lagen aktiv sind. Auch in der Jahresphänologie zeigen sich leichte Unterschiede: Hohe Aktivitäten treten bereits ab Juni, also außerhalb des herbstlichen Zuggeschehens, auf. Dies ist bei der Planung von Windparkprojekten in Bergwäldern zu berücksichtigen. Abschaltalgorithmen können nicht ohne weitere Anpassungen uneingeschränkt aus Tieflandstudien übernommen werden. Vorläufig ist aufgrund teilweise abweichender Aktivitätsmuster die Anwendung des ProBat-Tools an Bergwaldstandorten kritisch zu sehen. Da in den Bergwaldgebieten der Steiermark zu einem Großteil Windenergieanlagen mit niedrigen Rotor-Boden-Abständen zum Einsatz kommen, sollten zur Kontrolle der Wirksamkeit der Abschaltalgorithmen fallweise Schlagopfersuchen ab zumindest Juni durchgeführt werden. Zusätzliche Untersuchungen wären wünschenswert, um die Evidenzbasis zu systematischen Unterschieden zwischen Bergwald-, Tiefland- und anderen Gebieten zu erweitern. 


\section{Summary}

The Austrian climate and energy strategy plans a massive expansion of wind energy production. In Styria, most of the planned sites for wind farms are in montane forests. Studies on the intersections of bat activity and wind energy production have been conducted mostly at lowland sites. Recent studies in the Alps show surprisingly high bat activity at high elevations. As data on bat activity at nacelle height is virtually absent for Austrian montane forests, the present study aims to close this gap.

Bat activity was studied at five wind farms situated in Styrian montane spruce forests $(1,200-1,700 \mathrm{~m}$ a.s.1.) by using batcorders (ecoObs) in nacelles from at least May to mid-September. We recorded bat calls at all sites. In addition, wind speed was measured at four study sites and air temperature at two study sites at nacelle height. All data were collected in the context of planned wind energy projects. All study sites have a low rotor-ground distance of up to $50 \mathrm{~m}$.

Eight species were detected in the monitored wind park sites. The recorded species included bats from local populations like Pipistrellus pipistrellus and Eptesicus nilssonii as well as long-distance migratory species like Nyctalus noctula, Vespertilio murinus and $P$. nathusii. We recorded calls from the genus Myotis at a single study site. 85.2 to $97.4 \%$ of recordings were assigned to Nyctaloids and 2.5 to $14.7 \%$ to Pipistrelloids.

Variability in the number of recordings was very high between study sites, between years within study sites, and between wind turbines within the same wind park. Bat activity varied substantially not just between months, but also between days. We measured the highest activities of bats from June to September. In general, April and May featured a low acoustic activity of bats; at two sites relatively high activities were recorded still in October. The high variability underscores both the importance of multi-annual nacelle monitoring studies and of several sampled nacelles within a wind farm.

Bat activity was recorded at wind speeds of up to 6.5 to $15 \mathrm{~m} / \mathrm{s}$. As expected, bat activity decreased with increasing wind speeds, but this decrease varied strongly between sites and within sites between years. At some sites, bats were still active at freezing point temperatures.

These results indicate that bats in montane forests may be encountered at higher wind speeds and lower temperatures than in the lowlands. Further differences appear in the annual phenology; high activity occurs as early as June, i.e. preceding bat autumn migration periods. These differences should be taken into consideration when planning wind farms in montane forests. Curtailment algorithms require critical assessment if inferred from studies conducted at lowland sites. Due to differing activity patterns the application of the ProBat-tool is not recommended for the time being. As a result of the low rotor-ground distance of most of the wind 
energy plants in montane forests in Styria there should be case-to-case evaluations of the effectiveness of curtailment algorithms by means of fatality searches beginning at least in June. Further studies are needed to increase the evidence of systematic differences between montane forests, lowlands and other study sites.

\subsection{Einleitung}

Aus vielen europäischen Ländern sowie aus den USA sind Kollisionen von Fledermäusen mit Windenergieanlagen in teilweise erheblichem Umfang bekannt (Arnett et al. 2008; Brinkmann et al. 2011; Rydell et al. 2012; Santos et al. 2013; Voigt et al. 2015; Dürr 2019). Da Fledermäuse eine geringe Reproduktionsrate aufweisen, können größere Individuenverluste, die über die normale Sterblichkeit hinausgehen, nur schwer ausgeglichen werden (Kugelschafter 2013; Zahn et al. 2014; Pfalzer 2017; Lindemann et al. 2018). Aufgrund der Einstufung aller heimischen Fledermäuse in Anhang IV der Fauna-Flora-Habitat-Richtlinie (FFHRL) sind die Belange des strengen Artenschutzes (insbesondere das Tötungsverbot) bei der Planung und beim Betrieb von Windenergieanlagen zu berücksichtigen.

In Österreich hat sich die Bundesregierung zum Ziel gesetzt, bis zum Jahr $2030100 \%$ des Gesamtstromverbrauchs aus erneuerbarer Energie zu erzeugen. Um dieses Ziel zu erreichen, ist ein Ausbau aller erneuerbaren Energieträger notwendig. Die Windkraft stellt dabei einen wesentlichen Aspekt dar (BMNT und BMVIT 2018). Ende 2018 waren in Österreich 1313 Windenergieanlagen (WEA) in Betrieb. Rund 66 weitere Anlagen sind alleine für das Jahr 2019 in Planung (www.igwindkraft.at, Stand Januar 2019). Die Klima- und Energiestrategie des österreichischen Bundeslandes Steiermark (Amt der Steiermärkischen Landesregierung 2017) sieht einen Anteil von $40 \%$ an erneuerbarer Energie bis 2030 vor. Das bedeutet einen Ausbau der Windkraft in der Steiermark von 0,8 PJ (Stand 2015) auf 4,5 PJ, also um mehr als das Fünffache. Abgesehen von Repowering und der Errichtung bereits genehmigter bzw. in Genehmigung stehender Projekte ist dafür auch die Nutzung des vorhandenen Restpotenzials an Fläche nötig. Die geplanten Standorte für den Ausbau befinden sich hauptsächlich in Bergwaldgebieten. Darunter sind Wälder von der submontanen Stufe bis zur Baumgrenze, beginnend ab einer Höhe von $600 \mathrm{~m}$ bis zu $2000 \mathrm{~m}$ über NN zu verstehen. Die dominierende Baumart in den steirischen Bergwäldern ist die Fichte. Als weitere Baumarten sind Tanne, Kiefer, Lärche, Buche und Berg-Ahorn vertreten.

Bisherige Studien zum Thema Fledermausaktivität und Windkraft wurden vor allem im Tiefland oder in Mittelgebirgslagen durchgeführt (Brinkmann et al. 2011; Behr et al. 2015, 2018; Reichenbach et al. 2015; Hurst et al. 2016). Es gibt noch wenige Studien zur Fledermausaktivität aus höheren Lagen. So haben Zingg und Bontadina (2016) am Jungfrau Joch in den Schweizer Alpen auf einer Höhe von mehr als 3000 m über NN acht Fledermausarten nachgewiesen. 
Studien zum Thema Fledermauszug in den Alpen Österreichs; (Widerin und Reiter 2017, 2018) konnten teils beträchtliche Fledermausaktivität zur Zugzeit in Höhen bis zu $2500 \mathrm{~m}$ und auch über $3000 \mathrm{~m}$ über NN nachweisen. In einem Dauermonitoring am Hohen Sonnblick auf $3106 \mathrm{~m}$ über NN wurde festgestellt, dass Fledermäuse von Mitte April bis Mitte September aktiv waren, zum Teil auch bei Windgeschwindigkeiten von bis zu $12,2 \mathrm{~m} / \mathrm{s}$ und Temperaturen von bis $\mathrm{zu}-2,1^{\circ} \mathrm{C}$ (Widerin und Reiter 2018). Auch Bontadina et al. (2014) konnten eine regelmäßige Nutzung der Alpen durch Fledermäuse zeigen. Über Zugrouten von Fledermäusen im Alpenraum ist jedoch sowohl auf regionaler als auch auf überregionaler Ebene noch sehr wenig bekannt. Gerade für wandernde Fledermäuse wird das Kollisionsrisiko an Windenergieanlagen als ein kritischer Gefährdungsfaktor eingestuft (Voigt et al. 2012; Lehnert et al. 2014).

Diese Ergebnisse deuten darauf hin, dass die Fledermausaktivität in höheren Lagen bislang noch nicht ausreichend genug untersucht wurde. Insbesondere aus den Bergwäldern Österreichs liegen bis jetzt noch keine publizierten Daten zur Fledermausaktivität in Gondelhöhe vor. Ziel des vorliegenden Kapitels ist es, den Wissensstand darüber zu verbessern und Konsequenzen für die Bewertung von Windenergieanlagen in Bergwaldgebieten abzuleiten. Folgende Fragen sollen beantwortet werden: Welche Arten treten in Gondelhöhe in Bergwäldern der Steiermark in Höhen von 1200-1700 m über NN auf? Wie sieht die Phänologie über den Jahresverlauf aus, d. h., in welchen Monaten sind höhere Aktivitäten zu verzeichnen? Wie beeinflussen Windgeschwindigkeit und Temperatur die Fledermausaktivität in Bergwäldern?

\subsection{Material und Methoden}

\subsubsection{Standorte}

Es wurde an fünf verschiedenen Standorten in Bergwäldern der Steiermark, Österreich, an bestehenden Windenergieanlagen in Gondelhöhe die Fledermausaktivität gemessen. Die Standorte wurden nicht repräsentativ nach Zufallsprinzip ausgewählt, da es sich um Projekte im Rahmen von Windkraftplanungen handelte. Aus Gründen der Vertraulichkeit werden die Standorte anonymisiert aufgeführt. Dauer und Anzahl der beprobten Anlagen unterscheiden sich aufgrund von Vorgaben der jeweiligen Projektgenehmigungen. In den kleineren Windparks (Standorte 1, 2, 3 und 5) wurde je eine Gondel beprobt, an Standort 4 hingegen im Zuge der Erweiterung dieses Windparks drei von elf neu errichteten Anlagen (Tab. 6.1). Zwei der Windparks befanden sich im Wald, drei lagen direkt am Waldrand. Vorherrschende Baumart an allen Standorten war die Fichte, in geringen Anteilen war auch Lärche beigemischt (Tab. 6.1). Laubbäume wie zum Beispiel Rotbuche und Bergahorn kamen vereinzelt vor und nicht in direkter Nähe der beprobten 
Tab.6.1 Windparkstandorte nach Lage Wald/Waldrand, Höhe über NN, Anzahl beprobter Anlagen, Anzahl WEA im gesamten Windpark sowie Nabenhöhe und Rotordurchmesser der beprobten Anlagen

Tab. 6.1 Wind energy sites by position forest/forest edge, elevation above sea level, number of sampled wind turbines, size of wind farm, hub height and hub diameter

\begin{tabular}{l|l|l|l|l|l|l}
\hline Standort & Lage & $\begin{array}{l}\text { Höhe über } \\
\text { NN }[\mathrm{m}]\end{array}$ & $\begin{array}{l}\text { Anzahl } \\
\text { beprobte } \\
\text { Anlagen }\end{array}$ & $\begin{array}{l}\text { Anzahl } \\
\text { WEA im } \\
\text { Windpark }\end{array}$ & $\begin{array}{l}\text { Nabenhöhe } \\
{[\mathrm{m}]}\end{array}$ & $\begin{array}{l}\text { Rotordurch- } \\
\text { messer }[\mathrm{m}]\end{array}$ \\
\hline 1 & Waldrand & 1600 & 1 & 5 & 64 & 70 \\
\hline 2 & Wald & 1200 & 1 & 4 & 85 & 70 \\
\hline 3 & Waldrand & 1700 & 1 & 2 & 65 & 80 \\
\hline 4 & Waldrand & 1450 & 3 & 21 & 85 & 71 \\
\hline 5 & Wald & 1400 & 1 & 6 & 90 & 112 \\
\hline
\end{tabular}

Anlagen. An allen Standorten beträgt der Rotor-Boden-Abstand maximal $50 \mathrm{~m}$, bei dreien (Standorte 1, 3 und 5) beträgt er rund $30 \mathrm{~m}$.

\subsubsection{Erfassungszeitraum}

Die Untersuchungen wurden im Zeitraum von 2013 (Standort 1) bis 2018 (Standort 5) durchgeführt (Tab. 6.2). Die Standorte 1 und 2 wurden für je eine Saison beprobt (Windparkerweiterung sowie Gondelmonitoring nach Neuerrichtung eines Windparks für ein Jahr), Standort 3 für 1,5 Jahre (Repowering-Projekt) und die Standorte 4 und 5 für je zwei Saisonen (Gondelmonitoring nach Erweiterung eines Windparks bzw. Neuerrichtung), wobei bei Standort 5 die zweite Saison erst 2019 durchgeführt wird und daher hier nicht berücksichtigt werden kann. Bei den Standorten 1 bis 3 wurde nach Absprache mit der Behörde das Monitoring über zwei Kalenderjahre aufgeteilt: Es wurde im Spätsommer bzw. Herbst mit dem Monitoring begonnen, über den Winter ausgesetzt und dann im Folgejahr die Datenerfassung für Frühjahr und Sommer nachgeholt. Der Erfassungszeitraum lag an allen Standorten zumindest zwischen Mai und Mitte September. An den Standorten 2, 3 und teilweise 4 (nur im Jahr 2015 an zwei der drei beprobten Anlagen) wurden ab Spätsommer die Aufnahmezeiten in die Nachmittagsstunden ausgeweitet, um eventuellen Abendsegler-Tagzug erfassen zu können. Teilweise kam es zu Geräteausfällen aufgrund von technischen Problemen. Besonders davon betroffen war eine Anlage an Standort 4; hier musste aufgrund eines beschädigten Steuermoduls im Jahr 2015 das Gerät repariert werden, und es kam insgesamt zu einem zweimonatigen Datenausfall, der sich im darauffolgenden Jahr wiederholte.

Es wurden Batcorder 2 und 3 (ecoObs) für das Monitoring verwendet mit den Standardeinstellungen nach RENEBAT I: Quality 20, Threshold -36 dB, Posttrigger $200 \mathrm{~ms}$ und Critical Frequency $16 \mathrm{kHz}$. Vereinzelt wurde die Empfindlichkeit aufgrund übermäßiger Störgeräusche auf $-30 \mathrm{~dB}$ herabgesetzt; dies bedeutet 
Tab.6.2 Windparkstandorte nach Erfassungszeitraum, Zahl der Aufnahmenächte, Ausfallszeiten ab fünf Nächten und Threshold

Tab.6.2 Wind energy sites by recording period, recording nights, periods of missing recordings $>5$ nights and recording threshold

\begin{tabular}{|c|c|c|c|c|}
\hline Standort & Erfassungszeitraum & Aufnahmenächte & $\begin{array}{l}\text { Ausfall } \\
\text { (ab 5 Nächte) }\end{array}$ & Threshold [dB] \\
\hline 1 & $\begin{array}{l}\text { 01.10.-31.10.2013 } \\
\text { 01.04.-30.09.2014 }\end{array}$ & $31+183=214$ & keine & -36 \\
\hline 2 & $\begin{array}{l}\text { 05.08.-20.10.2014 } \\
\text { 14.04.-18.08.2015 }\end{array}$ & $77+83=160$ & $\begin{array}{l}\text { 17.-29.04., } \\
\text { 13.05.-02.06., } \\
\text { 19.-23.07.2015 }\end{array}$ & $\begin{array}{l}-36(2014) \\
-30(2015)\end{array}$ \\
\hline 3 & $\begin{array}{l}\text { 24.07.-15.11.2015 } \\
01.04 .-30.10 .2016\end{array}$ & $107+149=256$ & $\begin{array}{l}\text { 13.-30.07., 21.08.- } \\
\text { 01.09., 18.-25.09, } \\
\text { 02.-22.10.2016 }\end{array}$ & -36 \\
\hline 4_A & $\begin{array}{l}\text { 13.04.-7.11.2015 } \\
\text { 31.03.-16.11.2016 }\end{array}$ & $183+221=404$ & $\begin{array}{l}\text { 25.-29.4., } \\
\text { 18.-29.05., } \\
\text { 01.-09.06.2015; } \\
\text { 21.-29.08.2016 }\end{array}$ & -30 \\
\hline 4_B & $\begin{array}{l}\text { 30.03.-7.11.2015 } \\
\text { 13.04.-16.11.2016 }\end{array}$ & $156+143=299$ & $\begin{array}{l}\text { 19.05.-24.07.2015; } \\
\text { 11.-18.05., } \\
\text { 21.07.-29.09., } \\
\text { 11.09.-05.10.2016 }\end{array}$ & $-36 /-30$ \\
\hline 4_C & $\begin{array}{l}\text { 30.03.-7.11.2015 } \\
\text { 31.03.-16.11.2016 }\end{array}$ & $218+208=426$ & $\begin{array}{l}\text { 22.05.-26.05.2015; } \\
\text { 15.05-25.05., } \\
\text { 4.06.-14.06.2016 }\end{array}$ & $\begin{array}{l}-36 \\
(2015 / 2016) /-30 \\
(2015)\end{array}$ \\
\hline 5 & 01.05.-15.09.2018 & 112 & $\begin{array}{l}\text { 17.-22.05., } \\
\text { 04.-13.07.2018 }\end{array}$ & -36 \\
\hline
\end{tabular}

nach Tests von ecoObs, dass ca. 30-40\% weniger Aufnahmen im Vergleich $\mathrm{zu}-36 \mathrm{~dB}$ registriert werden. An Standort 1 wurde im Herbst 2013 mit einer Posttrigger-Einstellung von $400 \mathrm{~ms}$ beprobt, die Daten dieses Monats wurden später mithilfe der bcAdmin-Funktion ,virtual splitting“ auf 200 ms umgerechnet. Die Mikrofone wurden in den Gondeln jeweils mit Ausrichtung nach unten angebracht. Eine Ausnahme stellte Standort 3 dar; hier erfolgte die Messung aufgrund von Platzproblemen mittels Waldbox an der Außenwand der Gondel. Alle Mikrofone wurden vor dem ersten Einsatz sowie jährlich in den Wintermonaten zwischen Untersuchungsjahren kalibriert.

\subsubsection{Auswertung}

Als Auswertungsprogramme kamen bcAdmin und die automatische Rufbestimmungssoftware batIdent (beide ecoObs) zum Einsatz. Die automatisierten Bestimmungen wurden in allen Fällen manuell überprüft und bei Bedarf mittels bcAnalyze 3 Light (ecoObs) nachbestimmt. Ebenso wurden alle Aufnahmen 
geprüft, in denen auf automatisiertem Weg keine Fledermausrufe identifiziert wurden. Bei der Zuordnung der Aufnahmen der akustischen Dauererfassung wurden Aufnahmen ähnlich rufender Arten, die sich schwer oder nicht auf Artniveau bestimmen lassen, zu Rufgruppen zusammengefasst. So wurden alle Aufnahmen von Weißrand- und Rauhautfledermaus ohne Vorliegen von Sozialrufen dem Artenpaar zugeordnet. Die verwendeten Kurzbezeichnungen der Arten und Gruppen sind Tab. 6.3 zu entnehmen.

Es ist $\mathrm{zu}$ berücksichtigen, dass akustische Erfassungen nicht in allen Arten(gruppen) den gleichen Erfassungsgrad erreichen. Leise rufende Arten wie z. B. jene der Gattung Plecotus sind in akustischen Erfassungen generell unterrepräsentiert (Ahlen und Baggøe 1999). Die akustische Aktivität kann daher nicht als artübergreifendes absolutes Aktivitätsmaß gesehen werden, sondern als relatives Maß innerhalb einer Art bzw. Artengruppe mit ähnlichen Rufcharakteristika (Runkel et al. 2018).

Tab.6.3 Bezeichnungen für die Zuordnung der Aufnahmen zu bestimmten Arten bzw. Artengruppen mit ähnlichen Rufcharakteristika. (Nach Hurst et al. 2016)

Tab.6.3 Labeling for call classification to specific species and/or call groups with similar call characteristics. (Following Hurst et al. 2016)

\begin{tabular}{|c|c|c|}
\hline Bezeichnung & Kürzel & (Mögliche) Arten \\
\hline Pipistrellus pipistrellus & Ppip & Zwergfledermaus \\
\hline Pipistrellus pygmaeus & Ppyg & Mückenfledermaus \\
\hline Nyctalus noctula & Nnoc & Abendsegler \\
\hline Vespertilio murinus & Vmur & Zweifarbfledermaus \\
\hline Eptesicus nilssonii & Enil & Nordfledermaus \\
\hline Eptesicus serotinus & Eser & Breitflügelfledermaus \\
\hline Pipistrellus nathusii & Pnat & Rauhautfledermaus \\
\hline Pipistrellus nathusii/kuhlii & Pmid & $\begin{array}{l}\text { Rauhautfledermaus (P. nathusii), } \\
\text { Weißrandfledermaus (P. kuhlii) }\end{array}$ \\
\hline Phoch & Phoch & $\begin{array}{l}\text { Zwergfledermaus ( } P \text {. pipistrellus), Mückenfleder- } \\
\text { maus (P. pygmaeus) }\end{array}$ \\
\hline Nyctaloid & Nyctaloid & $\begin{array}{l}\text { Abendsegler (Nyctalus noctula), Kleinabend- } \\
\text { segler (N. leisleri), Zweifarbfledermaus } \\
\text { (Vespertilio murinus), Nordfledermaus (Eptesicus } \\
\text { nilssonii), Breitflügelfledermaus (E. serotinus), } \\
\text { Bulldoggfledermaus (Tadarida teniotis) }\end{array}$ \\
\hline Nyctaloid ,mittel““ & Nycmi & $\begin{array}{l}\text { Breitflügelfledermaus (E. serotinus), Zweifarb- } \\
\text { fledermaus (V. murinus), Kleinabendsegler ( } N \text {. } \\
\text { leisleri) }\end{array}$ \\
\hline Myotis sp. & Msp & Arten aus der Gattung Myotis \\
\hline Spec & Spec & $\begin{array}{l}\text { Aufnahmen von Fledermäusen, die keiner Art } \\
\text { zugeordnet werden können }\end{array}$ \\
\hline
\end{tabular}


Als Maß für die Fledermausaktivität wurde die Zahl der Aufnahmen verwendet. Für die Korrelationen der Fledermausaktivität mit den Witterungsparametern Windgeschwindigkeit und Temperatur wurden 10-min-Intervalle mit Aktivität verwendet, die Korrelation erfolgte mit bcAdmin (ecoObs).

\subsubsection{Witterungsdaten}

Die Witterungsdaten (Windgeschwindigkeit und Temperatur) wurden von den Anlagenbetreibern bereitgestellt. Die Windgeschwindigkeitsdaten wurden in Gondelhöhe mit einem Anemometer in 10-min-Intervallen aufgenommen. Die Temperaturdaten - falls vorhanden - wurden ebenfalls in 10-min-Intervallen in Gondelhöhe erhoben. Es lagen nur für die Standorte 4 und 5 Temperaturdaten vor. Für Standort 3 wurde vom Anlagenbetreiber keine Erlaubnis für die Verwendung der Witterungsdaten erteilt, daher beschränken sich die Auswertungen zu diesem Standort auf die Fledermausdaten.

\subsection{Ergebnisse}

\subsubsection{Artenspektrum}

An den fünf Windparkstandorten wurden insgesamt mindestens acht Fledermausarten nachgewiesen, wobei nicht alle Arten an jedem Standort vorkamen (Tab. 6.4 und Tab. 6.5). Das Artenspektrum umfasste sowohl ortstreue Arten, z. B. Zwergfledermaus und Nordfledermaus, als auch typische Langstreckenzieher, z. B. Abendsegler, Zweifarbfledermaus und Rauhautfledermaus. An allen Standorten wurden zahlreiche, nicht näher bestimmbare Rufe der Gruppen Nyctaloid bzw. Nycmi registriert. Es gab außerdem einige wenige Rufe, die aufgrund ihrer schlechten Qualität bzw. uneindeutigen Rufcharakteristik nur als nicht weiter bestimmte Fledermaus (,Spec“) klassifiziert werden konnten ( $\mathrm{n}=202,0,7 \%$, Tab. 6.5). Nur an Standort 5 wurden zwei Aufnahmen aus der Gattung Myotis dokumentiert.

Innerhalb der Pipistrelloid-Gruppe konnten Zwergfledermaus, Mückenfledermaus und das Artenpaar Rauhaut/Weißrandfledermaus bestimmt werden. An Standort 5 wurden zudem Sozialrufe der Rauhautfledermaus aufgenommen. An den restlichen vier Standorten wurde der überwiegende Teil der Aufnahmen des Artenpaares Rauhaut/Weißrandfledermaus zur Zugzeit verzeichnet, es ist daher ebenfalls von einem Vorkommen der Rauhautfledermaus auszugehen. Rufaufnahmen der Mückenfledermaus waren überall selten $(\mathrm{n}=33,0,1 \%)$, an Standort 5 fehlten sie gänzlich. Die Zwergfledermaus $(n=948$, 3,3 \%) konnte an allen Standorten mit Ausnahme von Standort 3 nachgewiesen werden. Generell trat die Zwergfledermaus an allen Windparkstandorten gehäuft von Juni bis Mitte August 
Tab. 6.4 Artenspektrum pro Untersuchungsstandort

Tab. 6.4 Recorded species/ call groups for each study site park

\begin{tabular}{l|l|l|l|l|l}
\hline \multirow{2}{*}{ Arten } & \multicolumn{5}{|l}{ Standort } \\
\cline { 2 - 7 } & $\mathbf{1}$ & $\mathbf{2}$ & $\mathbf{3}$ & $\mathbf{4}$ & $\mathbf{5}$ \\
\hline Pipistrelloid & & & & & \\
\hline Pipistrellus pipistrellus & $\mathrm{x}$ & $\mathrm{x}$ & & $\mathrm{x}$ & $\mathrm{x}$ \\
\hline Pipistrellus pygmaeus & $\mathrm{x}$ & $\mathrm{x}$ & $\mathrm{x}$ & $\mathrm{x}$ & \\
\hline Pipistrellus nathusii & & & & & $\mathrm{x}$ \\
\hline Pipistrellus nathusii/kuhlii & $\mathrm{x}$ & $\mathrm{x}$ & $\mathrm{x}$ & $\mathrm{x}$ & $\mathrm{x}$ \\
\hline Nyctaloid & & & & & \\
\hline Nyctalus noctula & $\mathrm{x}$ & $\mathrm{x}$ & $\mathrm{x}$ & $\mathrm{x}$ & $\mathrm{x}$ \\
\hline Vespertilio murinus & $\mathrm{x}$ & $\mathrm{x}$ & $\mathrm{x}$ & $\mathrm{x}$ & $\mathrm{x}$ \\
\hline Eptesicus nilssonii & $\mathrm{x}$ & $\mathrm{x}$ & $\mathrm{x}$ & $\mathrm{x}$ & $\mathrm{x}$ \\
\hline Eptesicus serotinus & & $\mathrm{x}$ & $\mathrm{x}$ & $\mathrm{x}$ & $\mathrm{x}$ \\
\hline Nycmi & $\mathrm{x}$ & $\mathrm{x}$ & $\mathrm{x}$ & $\mathrm{x}$ & $\mathrm{x}$ \\
\hline Myotis & & & & & \\
\hline Myotis sp. & & & & & $\mathrm{x}$ \\
\hline
\end{tabular}

auf, Aufnahmen wurden jedoch auch im September und teilweise Oktober verzeichnet. Die wenigen Aufnahmen der Mückenfledermaus wurden alle im Mai und August getätigt.

Der Großteil der Aufnahmen der Gruppe Nyctaloid ( $\mathrm{n}=26.467,92,1 \%)$ wurde nicht auf Artniveau bestimmt, es können daher keine Vergleiche zu den Häufigkeiten der nachgewiesenen Arten getroffen werden. In allen Windparks wurden Abendsegler, Zweifarbfledermaus und Nordfledermaus nachgewiesen; die Breitflügelfledermaus fehlte an Standort 1. An Standort 3 wurden Sozialrufe der Zweifarbfledermaus aufgezeichnet. An Standort 5 konnten sehr viele Rufe der Nyctaloid-Gruppe dem Abendsegler zugeordnet werden, diese traten dort gehäuft von Ende Juli bis Mitte September auf. Ein ähnliches Bild der Phänologie des Abendseglers zeigte sich auch an den anderen untersuchten Standorten: An den drei Standorten in Windpark 4 traten Aufnahmen des Abendseglers von Juni bis September auf, an Standort 2 mit einer deutlichen Häufung im September und Oktober. In keinem der drei Windparks, in denen die Aufnahmezeiten ab Spätsommer ausgeweitet wurden, konnten Hinweise auf einen Abendsegler-Tagzug festgestellt werden. Wie beim Abendsegler wurden auch bei der Zweifarbfledermaus vor allem Aufnahmen zur Zugzeit registriert, jedoch auch in den Sommermonaten ab Juni. Die Nordfledermaus wurde meist außerhalb der Zugzeit festgestellt, z. B. an Standort 4 im Juni und Juli, mit vereinzelten Aufnahmen noch im August. Die drei beprobten Anlagen in Windpark 4 zeigten Standortunterschiede der Nordfledermaus in der Raumnutzung: An 4_A und 4_C wurden Nordfledermäuse im Jahr 2015 und 2016 von Juni bis August nachgewiesen, an 4_B jedoch nur Juni und Juli. Generell wurden die meisten Aufzeichnungen der Nordfledermaus von Juni bis August registriert. Aufnahmen der Breitflügelfledermaus 


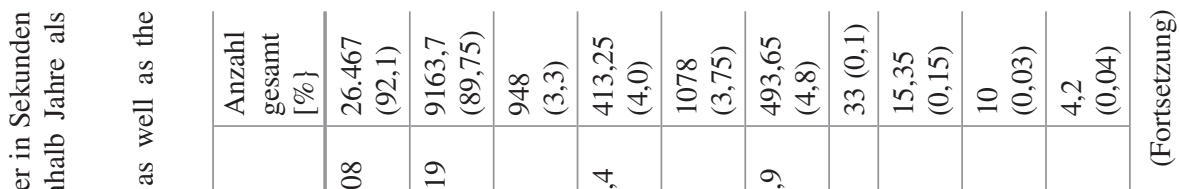

离.

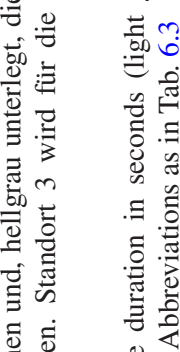

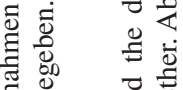

艺

矛 $\quad$ :

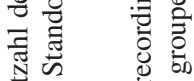

苛苛䒕

ปั:

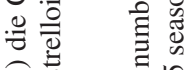

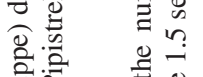

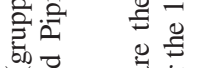

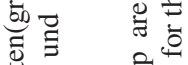

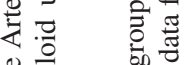

造 क

灵合

苂

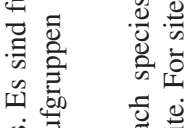

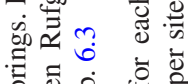

:

ह

을

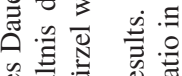

约得语

D ए

잉ㅁㅇ

司

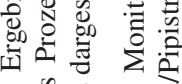

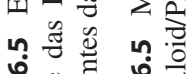

ํ.

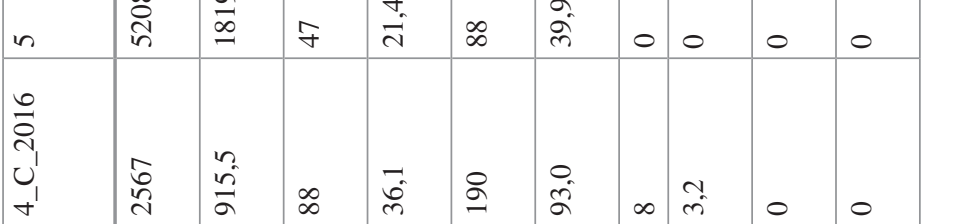

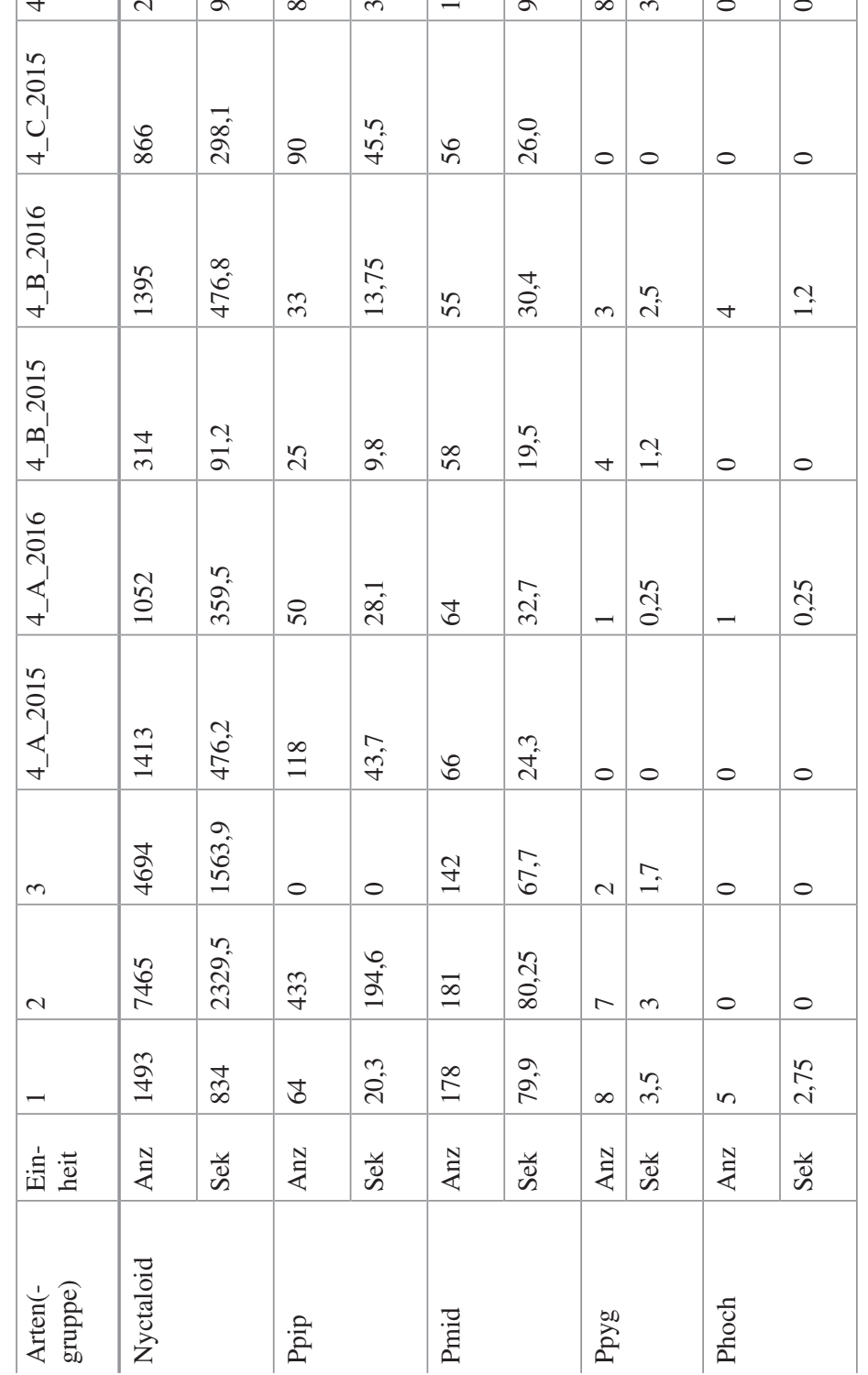


132

S. Huemer und B. Komposch

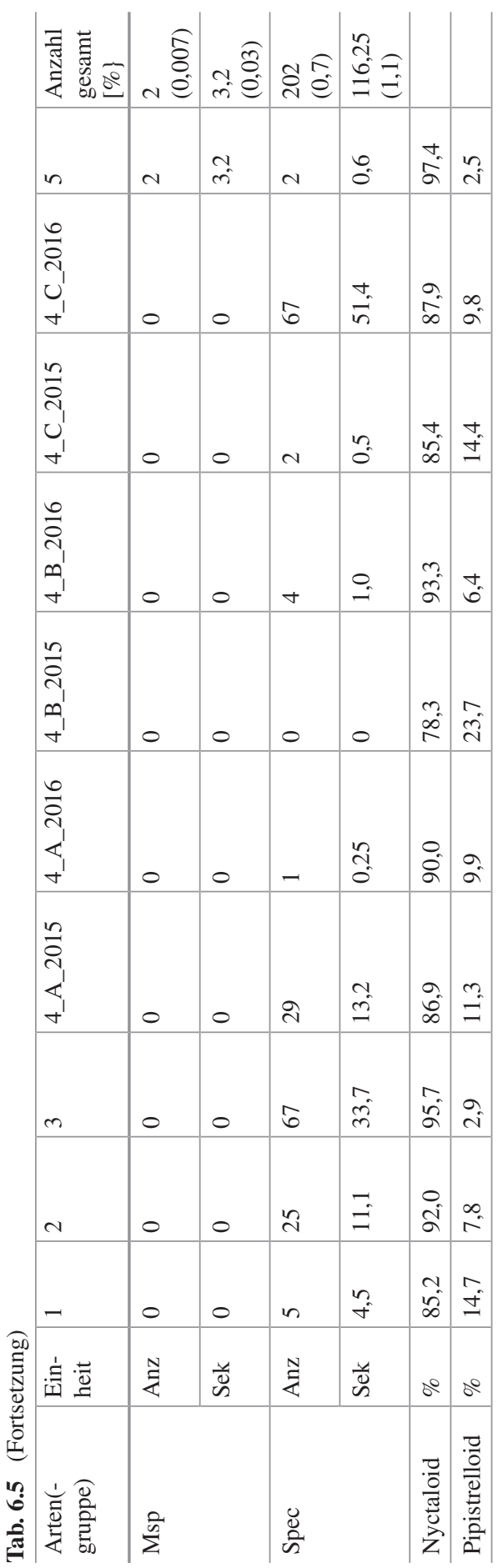


wurden ebenfalls von Juni bis Anfang August, an Standort 3 auch im September nachgewiesen.

Es stammten je nach Standort rund 85 bis $97 \%$ der Aufnahmen von Vertretern der Gruppe Nyctaloid. Nur an Standort 4_B_2015 betrug dieser Wert rund $78 \%$. Dieser Standort ist jedoch aufgrund des zweimonatigen Datenausfalls im Frühsommer als nicht repräsentativ zu betrachten. Die höchsten Werte mit 95,7 \% bzw. 97,4 \% traten an den Standorten 2 und 5 auf. Dementsprechend betrug der Anteil an Aufnahmen der Gruppe Pipistrelloid je nach Standort zwischen 2,5 und 14,7 \%. Vernachlässigbar war der Anteil der Aufnahmen der Gattung Myotis.

\subsubsection{Aktivitätslevel an den untersuchten Standorten}

Es wurde eine hohe Variabilität der Aufnahmenzahlen verzeichnet, nicht nur zwischen den fünf Standorten, sondern auch zwischen den Jahren der über zwei Saisonen untersuchten Standorte (Abb. 6.1). An den fünf untersuchten Standorten ragte Standort 2 mit 8096 Aufnahmen heraus, gefolgt von Standort 5 mit 5340 Aufnahmen; diese beiden Standorte stellen hinsichtlich der Höhenlage die tiefstgelegenen Untersuchungsflächen dar (Standort 2: rund 1200 m; Standort 4: rund $1400 \mathrm{~m}$ über NN). Weiterhin liegen nur diese beiden Standorte zur Gänze innerhalb eines Bergwaldes. Aber auch an Standort 3, der am Waldrand auf etwas über 1700 m liegt, wurden im Jahr 2016 an 149 Nächten 3680 Aufnahmen verzeichnet.

Die hohe Variabilität zwischen den Jahren ist an Standort 4, an dem drei Anlagen beprobt wurden, gut zu erkennen: So zeigte Standort 4_C im Jahr 2016 eine mehr als doppelt so hohe Fledermausaktivität als 2015, obwohl im Jahr 2016 weniger Aufnahmenächte vorlagen als 2015. An Standort 4_A hingegen wurde im Jahr 2016 weniger Aktivität als 2015 verzeichnet, trotz einer höheren Anzahl an Aufnahmenächten. Während bei 4_C der niedrigere Schwellenwert von $-30 \mathrm{~dB}$ ab Mitte August 2015 für die Differenz mitverantwortlich sein könnte, wurde 4_A immer mit den gleichen Parametern $(-30 \mathrm{~dB})$ beprobt. Nicht repräsentativ ist Standort 4_B: Die ungewöhnlich niedrige Zahl an Aufnahmen im Jahr 2015 ist auf einen zweimonatigen Geräteausfall von Ende Mai bis Ende Juli zurückzuführen. Im Jahr 2016 wurde gerade im Juni an dieser Anlage mehr als die Hälfte der Gesamtaktivität des Jahres erreicht.

\subsubsection{Jahreszeitliche Phänologie der Aktivität}

An allen Standorten war ein deutlicher Anstieg der Fledermausaktivität ab Juni zu verzeichnen (Abb. 6.2). Die höchsten Aktivitäten wurden generell in den Monaten Juni bis September gemessen. An zwei Standorten, 1 und 4_B_2016, war der Juni der Monat mit der höchsten Aktivität. Im Oktober wurden mit Ausnahme von Standort 1 und vor allem Standort 2 meist geringere Aktivitäten registriert. An Standort 3 waren die Geräte im ersten Untersuchungsjahr bis Mitte November 2015 im Einsatz. In diesen 15 Novembertagen wurden auf rund 1700 m Höhe 
Anzahl Aufnahmen/Standort/Jahr

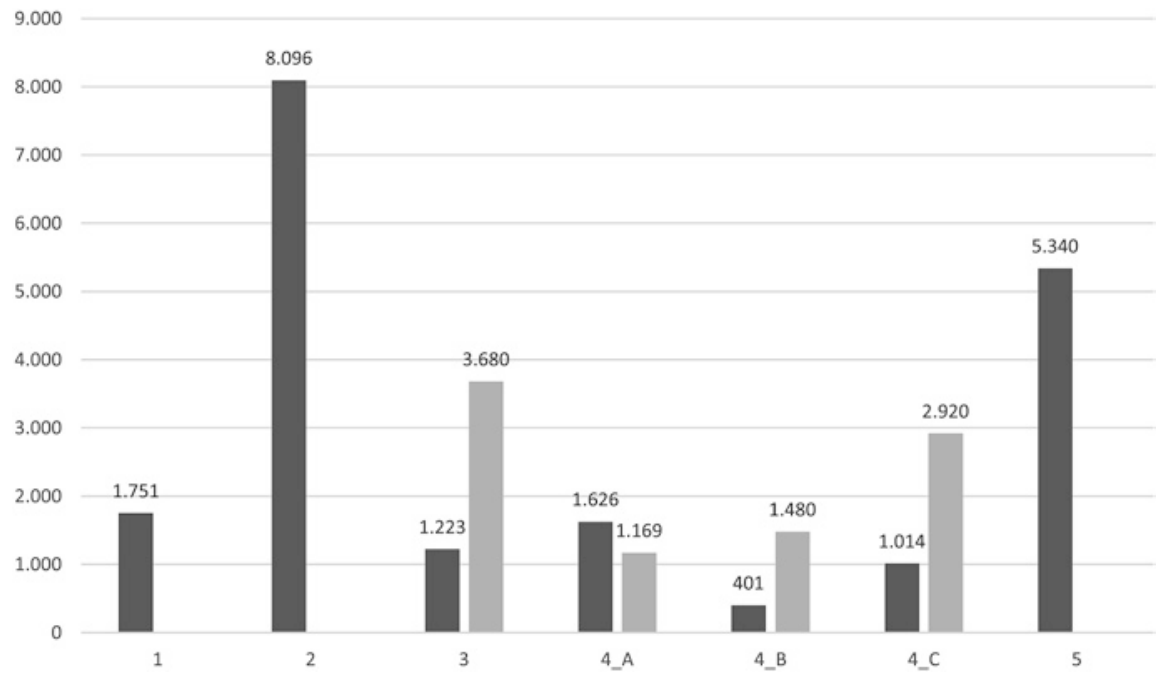

Abb. 6.1 Anzahl der Aufnahmen pro Standort pro Jahr. Standorte, an denen das Monitoring auf zwei Kalenderjahre aufgeteilt wurde, insgesamt jedoch nicht länger als eine Saison lief, werden gemeinsam dargestellt (Standort 1 und 2). Dunkelgrauer Balken=Jahr 1, hellgrauer Balken $=$ Jahr 2. Anmerkung: Jahr 1/2 und Anzahl der Aufnahmenächte pro Standort variieren zwischen den Standorten (siehe Tab. 6.2). * = Standorte mit über die Gesamtzeit (4_A) bzw. teilweise (2, 4_B, 4_C) reduzierter Empfindlichkeit (Threshold -30 dB) (siehe auch Tab. 6.2)

Fig. 6.1 Number of recordings per site per year. Sites which were monitored for one season but over two calendar years are shown together (site 1 and 2). Dark grey bar=year 1, light grey bar $=$ year 2. Note: Year $1 / 2$ and the number of recording nights per site differ between the sites (see tab. 2). * = sites with reduced sensitivity (threshold $-30 \mathrm{~dB}$ ) over the entire (4_A) or some part of the monitored time (2, 4_B, 4_C) (see Tab. 6.2)

über NN noch 56 Aufnahmen aufgezeichnet. An Standort 4 auf 1450 m Höhe über NN wurden die Geräte 2015 und 2016 ebenfalls erst im Laufe des Novembers abgebaut, es wurde jedoch in keinem der beiden Jahre Aktivität im November registriert. Im April und Mai wurden generell niedrige Aktivitäten dokumentiert. Bei den Standorten mit zweijährigen Monitorings ergab sich eine große Variabilität in der Aktivität zwischen den Jahren: So hatten die Standorte 4_A und 4_C nur sehr wenige Aufnahmen im September 2015, im darauffolgenden Jahr war dies aber der Monat mit der höchsten bzw. zweithöchsten Aktivität. Die Anzahl der beprobten Nächte war für beide Jahre gleich. An Standort 4_C wurde im Jahr 2015 mit unempfindlicheren Einstellungen $(-30 \mathrm{~dB})$ gemessen als 2016, was einen Einfluss haben könnte, da bei geringerem Schwellenwert weniger Aufnahmen registriert werden. An Standort 4_A waren die Einstellungen jedoch über beide Jahre identisch. Möglicherweise waren klimatische Faktoren ausschlaggebend, der September 2016 war sehr warm und trocken. Auch an Standort 3 
a

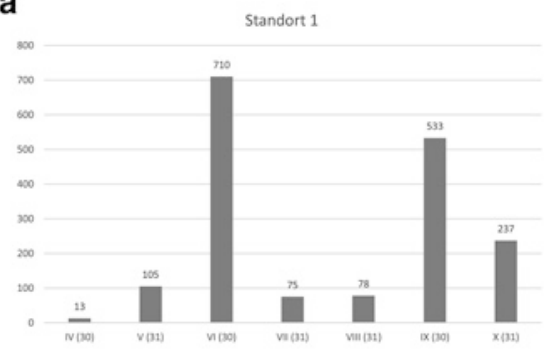

C

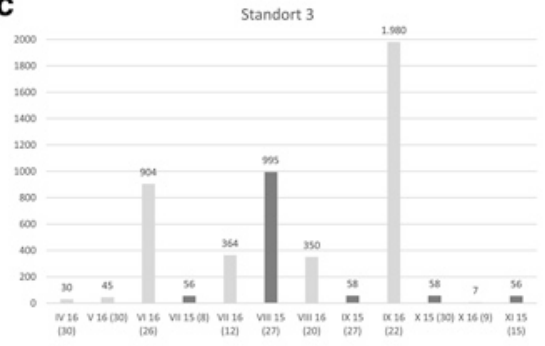

e

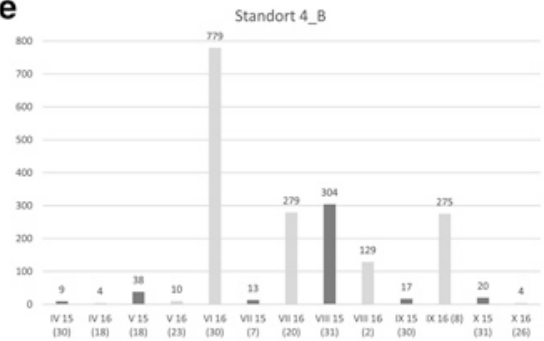

g

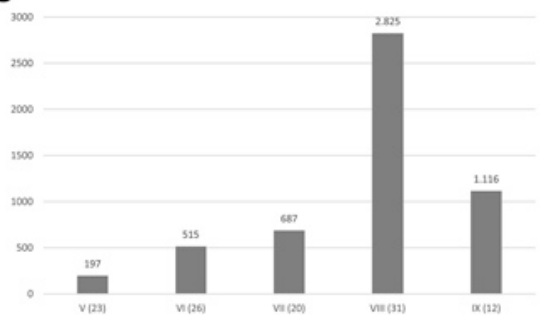

b

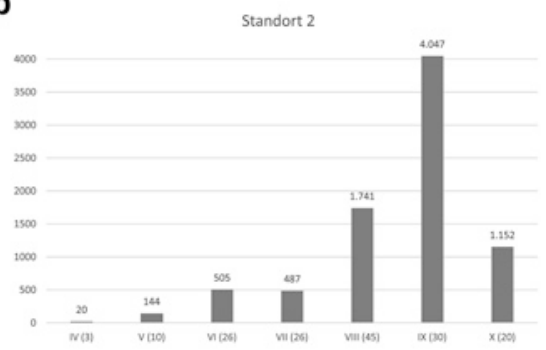

d

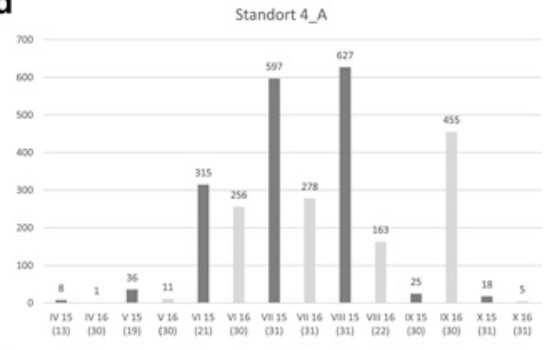

f

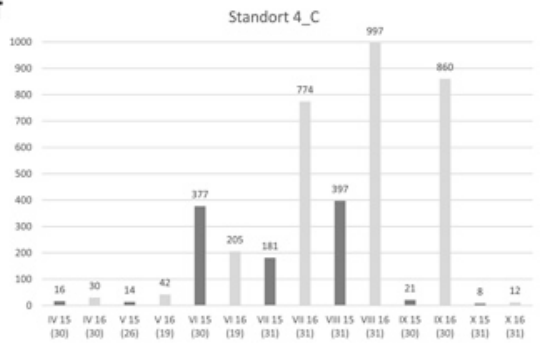

Abb. 6.2a-g Anzahl der Aufnahmen pro Monat pro Standort. Standorte, in denen das Monitoring auf zwei Kalenderjahre aufgeteilt wurde, insgesamt jedoch nicht länger als eine Saison lief, werden gemeinsam dargestellt (1 und 2). Monate in römischen Ziffern. In Klammer die Anzahl der Aufnahmenächte. Dunkelgraue Balken=Jahr 1, hellgraue Balken=Jahr 2. Zur besseren Darstellbarkeit sind die Grafiken unterschiedlich skaliert

Fig. 6.2a-g Number of recordings per month per site. Sites which were monitored for one season but over two calendar years are shown together (1 and 2). Months in Roman numerals. In parentheses the number of recording nights is shown. Dark grey bar=year 1 , light grey bar $=$ year 2 . For better visualisation the graphs are scaled differently 
wurde im September 2016 ein Vielfaches der Aufnahmenzahl von September 2015 (1980 versus 58) aufgenommen, bei gleichen Einstellungen und trotz weniger Aufnahmenächte im Jahr 2016. Standort 4_A und 4_C zeigten auch eine jeweils umgekehrte Variabilität von Aufnahmen im Juli bei jeweils gleicher Anzahl von Aufnahmenächten: Während an Standort 4_A im Juli 2015 mehr als die doppelte Menge an Aufnahmen im Vergleich zu 2016 registriert wurde, war die Aufnahmenzahl an Standort 4_C im Juli 2015 nur ein Viertel der Aufnahmenzahl von 2016. Die dokumentierte Fledermausaktivität schwankte nicht nur zwischen den einzelnen Monaten stark, sondern auch an einzelnen Tagen. So wurden an Standort 1 mehr als $80 \%$ der Fledermausaktivität im Juni an nur fünf Nächten aufgenommen, an Standort 2 wurden in einer Nacht im September 3037 Aufnahmen und damit der Großteil der Aktivität in diesem Monat aufgezeichnet.

\subsubsection{Aktivität in Abhängigkeit zu Windgeschwindigkeit und Temperatur}

Die höchsten Windgeschwindigkeiten, bei denen Fledermäuse noch aktiv waren, reichten an den unterschiedlichen Standorten von 6,5 bis 15 m/s (Spalte „Max.“, Tab. 6.6). Erwartungsgemäß nahm die Fledermausaktivität mit zunehmender Windgeschwindigkeit ab. Dieser Rückgang variierte jedoch von Standort zu Standort und auch innerhalb eines Standortes von Jahr zu Jahr. An Standort 1 wurden $95 \%$ der Fledermausaktivität bis $8 \mathrm{~m} / \mathrm{s}$ verzeichnet. An den Stand-

Tab. 6.6 Windgeschwindigkeiten und Minimumtemperaturen pro Standort. Max. = maximale Windgeschwindigkeit, bei der noch Fledermausaktivität gemessen wurde. $85 \%$ bzw.

$95 \%=$ Windgeschwindigkeiten der 85- und 95-Perzentile der 10-min-Intervalle mit Fledermausaktivität, $\mathrm{nA}=$ keine Angaben

Tab. 6.6 Wind speed and minimum temperature per site. Max.= maximum wind speed when bat activity was recorded and wind speeds of 85 and 95 percentiles of 10-min-intervals with recorded bat activity, $\mathrm{nA}=$ not available

\begin{tabular}{l|l|l|l|l}
\hline Standort & $\begin{array}{l}85 \% \\
{[\mathrm{~m} / \mathrm{s}]}\end{array}$ & $\begin{array}{l}95 \% \\
{[\mathrm{~m} / \mathrm{s}]}\end{array}$ & $\begin{array}{l}\text { Max } \\
{[\mathrm{m} / \mathrm{s}]}\end{array}$ & $\begin{array}{l}\text { Minimumtemperatur } \\
{\left[{ }^{\circ} \mathrm{C}\right]}\end{array}$ \\
\hline 1 & 6,0 & 8,0 & 15,0 & $\mathrm{nA}$ \\
\hline 2 & 5,0 & 6,5 & 12,0 & $\mathrm{nA}$ \\
\hline 4_A 2015 & 5,5 & 6,5 & 11,5 & 0 \\
\hline 4_B 2015 & 5,0 & 6,0 & 6,5 & 3 \\
\hline 4_C 2015 & 5,5 & 7,0 & 14,0 & $-1,0$ \\
\hline 4_A 2016 & 5,0 & 6,0 & 9,5 & 7 \\
\hline 4_B 2016 & 5,5 & 6,5 & 10,5 & 1 \\
\hline 4_C 2016 & 5,5 & 7,0 & 9,5 & 0 \\
\hline 5 & 5,0 & 6,5 & 14,0 & 11 \\
\hline
\end{tabular}


orten 4_A_2016 sowie 4_B_2015 wurden $95 \%$ der Aktivität hingegen bis $6 \mathrm{~m} / \mathrm{s}$ verzeichnet, in den beiden anderen Untersuchungsjahren an diesen Standorten (4_A_2015 und 4_B_2016) bei etwas höheren Windgeschwindigkeiten bis $6,5 \mathrm{~m} / \mathrm{s}$. Nur an Standort 4_C blieben diese Werte in beiden Jahren gleich; jeweils $85 \%$ der Fledermausaktivität fanden bis $5,5 \mathrm{~m} / \mathrm{s}$ statt und $95 \%$ bis $7,0 \mathrm{~m} / \mathrm{s}$. Bei den maximalen Windgeschwindigkeiten, bis zu denen Fledermäuse aktiv waren, gab es jedoch auch bei diesem Standort Unterschiede; im Jahr 2015 waren dies $14,0 \mathrm{~m} / \mathrm{s}$ versus $9,5 \mathrm{~m} / \mathrm{s}$ im Jahr 2016.

Von zwei Standorten liegen Temperaturdaten in Gondelhöhe vor. Sie zeigen, dass Fledermausaktivität teilweise bereits bei Temperaturen um den Gefrierpunkt verzeichnet wurde (Standort 4_A_2015 sowie 4_C_2015 und 2016). An Standort 4 wurden Fledermausrufe jedoch auch erst ab $7{ }^{\circ} \mathrm{C}$ registriert (4_A_2016). Standort 5 wich mit einem Beginn der Fledermausaktivität erst ab $11^{\circ} \mathrm{C}$ deutlich ab. Der Sommer 2018 war ein Hitzesommer, trotzdem sind diese Temperaturen für Nächte in dieser Höhenlage ungewöhnlich hoch; eventuell handelt es sich um einen systematischen Messfehler im SCADA-System der Windenergieanlage.

\subsection{Diskussion}

\subsubsection{Schlussfolgerungen zum Artenspektrum}

In Höhen zwischen $1200 \mathrm{~m}$ und $1700 \mathrm{~m}$ über NN wurden in Gondelhöhe mindestens acht Fledermausarten nachgewiesen. Im Artenspektrum waren die drei Langstreckenzieher Abendsegler, Zweifarbfledermaus und Rauhautfledermaus vertreten. Nordfledermaus, Zwergfledermaus und Breitflügelfledermaus sind lokalen Populationen zuzuordnen. Breitflügelfledermäuse wurden nicht häufig aufgenommen. Es liegen vorwiegend aus den Monaten Juni bis August, aber auch noch September Aufnahmen vor. Wahrscheinlich handelt es sich um Einzeltiere, die zum Jagen in höhere Lagen fliegen. Den Verfasserinnen ist zumindest ein (Männchen-)Quartier dieser Art auf fast 600 m Höhe über NN in der Steiermark bekannt. Von der Mückenfledermaus gab es vereinzelt Aufnahmen im Mai und August. Hier ist unklar, ob es sich um lokale oder ziehende Tiere handelt. Nach Dietz et al. (2016) sind von dieser Art kaum Langstreckenflüge in Mitteleuropa bekannt. Die Ergebnisse im Artenspektrum decken sich mit den Befunden von Widerin und Reiter (2018) sowie Zingg und Bontadina (2016), die in noch größeren Höhen von mehr als 3000 m über NN sechs bzw. acht Arten sowie ein fast identisches Artenspektrum nachweisen konnten. Jene beiden Studien stellten den ebenfalls zu den Langstreckenziehern gehörenden Kleinabendsegler fest. Dieser konnte in der vorliegenden Studie nicht nachgewiesen werden. Es könnte sich jedoch unter den Aufnahmen der Gruppen Nyctaloid und Nycmi auch diese schwer bestimmbare Art befinden. 
Sieben von den acht nachgewiesenen Arten gelten als kollisionsgefährdet (Hurst et al. 2015, 2016). Für wandernde Fledermäuse wird das Kollisionsrisiko an WEA als ein relevanter Gefährdungsfaktor eingestuft (z. B. Brinkmann et al. 2011; Voigt et al. 2012; Lehnert et al. 2014). Es sind jedoch auch lokal reproduzierende Arten von Kollisionen betroffen, wenn sie im freien Luftraum jagen oder durch WEA angelockt werden (Behr et al. 2015; Pfalzer 2017; Zahn et al. 2014). So sind Nordfledermäuse eher ortstreu (Dietz et al. 2016), mit Schlagopfern ist hier nach vor allem in den Mittelgebirgslagen im Spätsommer zu rechnen. Bei Zwergfledermäusen (sowie bei anderen Arten der Gattung Pipistrellus) wird angenommen, dass sie überwiegend an WEA in und bei Wäldern einem erhöhten Risiko ausgesetzt sind (Zahn et al. 2014). So wurden in einer Studie im Schwarzwald viele verunglückte Zwergfledermäuse speziell in der Schwärm- und Quartiererkundungsphase im August gefunden. Als Ursache hierfür wird das Erkundungsverhalten angenommen (Zahn et al. 2014). Bemerkenswert waren in der vorliegenden Untersuchung zwei Aufnahmen der Gattung Myotis an einem Standort in 1400 m Höhe über NN. In den Studien von Reichenbach et al. (2015) sowie Hurst et al. (2016) wurde die Gattung Myotis nur in Ausnahmefällen in dieser Höhe aufgenommen. Damit erwarten die Autoren dieser Studien auch keine erhöhte Schlaggefährdung für diese Gruppe. Eine Ausnahme könnten nach Hurst et al. (2016) jedoch Standorte darstellen, in denen die Rotorblätter nahe an die Waldoberkante reichen. Dies trifft in diesem Fall zu, da an Standort 5 der Rotor-Boden-Abstand nur $34 \mathrm{~m}$ betrug und die Rotoren damit bis knapp an die Baumkronen reichten.

In der vorliegenden Studie wurden Alpenfledermaus (Hypsugo savii), Langflügelfledermaus (Miniopterus schreibersii) und Bulldoggfledermaus (Tadarida teniotis) nicht nachgewiesen. Letztere könnte übersehen worden sein, da die CF-Einstellung für eine Erfassung dieser Art nicht ausreichend war. In Österreich wurde die Bulldogfledermaus bislang nur vereinzelt in Tirol festgestellt. Die nächstgelegenen Nachweise südlich von Österreich stammen aus Slowenien (Presetnik und Šalamun 2019). Wandernde Tiere können jedoch nicht komplett ausgeschlossen werden. Es wurden an allen Standorten Aufnahmen des Artenpaares Rauhaut-/Weißrandfledermaus getätigt, vereinzelt auch in den Sommermonaten außerhalb der Zugzeit, d. h., ein Vorkommen der Weißrandfledermaus kann ebenfalls nicht ausgeschlossen werden.

Die Zusammensetzung des Artenspektrums ist in der vorliegenden Untersuchung stark von der Gruppe Nyctaloid dominiert, mit 85-97,5\% gegenüber 2,5-15\% der Gruppe Pipistrelloid. Damit liegt der Anteil an Nyctaloiden höher als bei den RENEBAT-Studien mit rund 42-76\% Anteil an Nyctaloiden (Behr et al. 2018). 


\subsubsection{Schlussfolgerungen zu Fledermausaktivität und Phänologie}

In der vorliegenden Untersuchung konnten an einzelnen Standorten hohe Fledermausaktivitäten festgestellt werden. Es wurde zudem gezeigt, dass die Fledermausaktivität sowohl zwischen den Standorten als auch am selben Standort zwischen den Jahren im selben Kalendermonat stark variierte. Dies deckt sich mit den Befunden von Hurst et al. (2016) sowie Widerin und Reiter (2018). Verglichen mit anderen Studien wie z. B. Brinkmann et al. (2011) unterscheiden sich jedoch zum Teil die Aktivitätsmaxima. Während zum Beispiel Standort 1 in der vorliegenden Untersuchung im Juli und August eine auffallend geringe Aktivität aufwies, konnten Brinkmann et al. (2011) ein Aktivitätsmaximum von der zweiten Julihälfte bis zur ersten Septemberhälfte aufzeigen. Es ist zu vermuten, dass der kühle und regnerische Sommer im Jahr 2014 an Standort 1 Auswirkungen auf die Fledermausaktivität in diesen Monaten hatte. Im Gegensatz dazu steht an diesem Standort die sehr hohe Aktivität im Juni, also zu einer Zeit außerhalb des Zuggeschehens. Generell stieg die Fledermausaktivität an allen Standorten dieser Untersuchung ab Juni markant an, also früher als in tieferen Lagen (Brinkmann et al. 2011; Behr et al. 2015; Reichenbach et al. 2015; Hurst et al. 2016). Bei den Langstreckenziehern waren ein deutlicher Anstieg der Aktivität ab Spätsommer und eine vergleichsweise geringe Frühjahrsaktivität zu verzeichnen. Dieses Ergebnis findet sich auch in anderen Studien in tieferen und höheren Lagen wieder (Behr et al. 2018; Widerin und Reiter 2018).

An Standort 2 zeigt sich exemplarisch die hohe Schwankungsbreite an Aufnahmen pro Nacht. Der September sticht an diesem Standort als der Monat mit der höchsten Fledermausaktivität heraus (4047 Aufnahmen). Dies ist vor allem durch die Nacht vom 30. September 2014 bedingt, in der 3037 Aufnahmen verzeichnet wurden. Auch an anderen Tagen wurde vereinzelt eine sehr hohe Aktivität registriert: fast 800 Aufnahmen am 2. August 2015 sowie 559 Aufnahmen am 11. Oktober 2014. Eine ähnliche Häufung von Fledermausaktivität in einzelnen Nächten trat auch an Standort 1 auf, wo $80 \%$ der Aktivität im Juni an fünf Nächten aufgezeichnet wurden. Die Beobachtung, dass sich die Fledermausaktivität stark auf einzelne Tage konzentriert, unterstreicht die Notwendigkeit des Dauermonitorings bei Windkraftprojekten. Dies deckt sich auch mit dem aktuellen Kenntnisstand (Hurst et al. 2016; Behr et al. 2018). Eine weitere Konsequenz sollte aus der auch in dieser Fallstudie nachgewiesenen starken Variabilität der Fledermausaktivität zwischen den Jahren gezogen werden. Alleine aufgrund klimatischer Bedingungen kann die Aktivität in einem Monat von Jahr zu Jahr stark schwanken. Einjährige Gondelmonitorings können diese Variabilität nicht abbilden. Für Windenergieprojekte im Bergwaldlebensraum sollte deshalb ein Dauermonitoring mindestens zwei Jahre umfassen. Es stellt sich zusätzlich die Frage, ob in gewissen Zeiträumen (z. B. alle drei Jahre; Hurst et al. 2016) nicht eine Wiederholung der Messungen durchgeführt werden 
sollte, um über die Lebensdauer der Anlagen die Ergebnisse zu evaluieren. Die großen Unterschiede zwischen der Fledermausaktivität an den Standorten von Windpark 4 unterstreichen außerdem die Notwendigkeit von mehreren Gondelmonitoring-Standorten innerhalb eines Windparks. Die Fledermausaktivität kann kleinräumig stark variieren. Auch das Forschungsvorhaben RENEBAT III (Behr et al. 2018) stellte eine hohe Variabilität in der Aktivität innerhalb eines Windparks sowie in verschiedenen Jahren im selben Windpark fest.

\subsubsection{Schlussfolgerungen zu Aktivität in Abhängigkeit von Windgeschwindigkeit und Temperatur}

Wie erwartet war an allen Standorten die Fledermausaktivität stark abhängig von Windgeschwindigkeiten und Temperaturen. Die Aktivität ging mit zunehmenden Windgeschwindigkeiten sowie mit niedrigeren Temperaturen zurück. Die maximalen Windgeschwindigkeiten, bis zu denen Fledermausaktivität beobachtet wurde, schwankten pro Standort von 6,5-15 m/s. Großteils wurden noch $5 \%$ der Fledermausaktivität bei Windgeschwindigkeiten über $6,5 \mathrm{~m} / \mathrm{s}$ verzeichnet, an drei Standorten auch über 7 bzw. $8 \mathrm{~m} / \mathrm{s}$. An den drei beprobten Anlagen an Standort 4 zeigte sich eine Variabilität zwischen den Jahren, wobei zwei Standorte (4_A und 4_B) jeweils gegenläufige Tendenzen aufwiesen. Temperaturdaten waren nur von zwei Standorten vorhanden. Hier unterscheiden sich die Ergebnisse deutlich: Fledermäuse waren ab Temperaturen von $-1{ }^{\circ} \mathrm{C}$ bzw. $+11{ }^{\circ} \mathrm{C}$ aktiv. Ab einer Temperatur von rund $10^{\circ} \mathrm{C}$ stieg die Fledermausaktivität stark an. Jedoch wurden mit Ausnahme von einem Standort noch $5 \%$ Fledermausaktivität bereits unter $10{ }^{\circ} \mathrm{C}$ verzeichnet.

Vergleicht man diese ersten Ergebnisse mit Studien aus tieferen Lagen, so wurde bei Behr et al. (2011) im Forschungsvorhaben RENEBAT I gezeigt, dass $6 \%$ der Fledermausaktivität im Bereich von WEA Gondeln bei Windgeschwindigkeiten über $6 \mathrm{~m} / \mathrm{s}$ stattfanden, bei RENEBAT II (Behr et al. 2015) wurden $4 \%$ der Fledermausaktivität im Bereich von WEA Gondeln über $5 \mathrm{~m} / \mathrm{s}$ festgestellt (beide etwa 95-Perzentil). Hingegen wurden bei Hurst et al. (2016) noch $20 \%$ der 10-min-Intervalle mit Fledermausaktivität bei Windgeschwindigkeiten über $6 \mathrm{~m} / \mathrm{s}$ registriert; diese Untersuchung fand an Windmasten statt, nicht in bereits bestehenden Windparks. Hinsichtlich Temperatur wurde in tieferen Lagen häufig nur noch geringe Aktivität bei Temperaturen unter $10^{\circ} \mathrm{C}$ festgestellt (Brinkmann et al. 2011; Behr et al. 2015; Hurst et al. 2016). Betrachtet man die Studien aus dem Alpenraum, so konnten Widerin und Reiter (2017, 2018) Fledermausaktivität noch bei Windgeschwindigkeiten von 12,2 bzw. $13 \mathrm{~m} / \mathrm{s}$ und Temperaturen bis zu $-5,8^{\circ} \mathrm{C}$ zeigen. Auch Widerin und Jerabek (2014) wiesen Fledermausaktivität bei geringen Temperaturen in Höhenlagen nach.

Zusammen mit anderen Befunden aus den Alpen zeigen die vorliegenden Ergebnisse aus den Bergwäldern der Steiermark, dass Fledermäuse in höheren Lagen bei höheren Windgeschwindigkeiten und niederen Temperaturen aktiver 
sein können als in tieferen Lagen. Dies muss bei der Planung von Windparkprojekten in Bergwäldern berücksichtigt werden. Abschaltalgorithmen können nicht ohne entsprechende Anpassungen aus Tieflandstudien übernommen werden. Derzeit kommen für das erste Betriebsjahr von Windenergieanlagen im Bergwald meist die von RENEBAT empfohlenen Abschaltalgorithmen von $<6 \mathrm{~m} / \mathrm{s}$ und $>10^{\circ} \mathrm{C}$ zur Anwendung, bevor diese durch ein Gondelmonitoring adaptiert werden. In Abhängigkeit von den Ergebnissen der Voruntersuchungen sind diese Werte zu modifizieren. So fordert z. B. die Koordinationsstelle für Fledermausschutz- und -forschung in Österreich (KFFÖ) in ihrem Positionspapier (AG Fledermäuse und Windenergie 2014) als Temperaturwert für das Bergland $>8{ }^{\circ} \mathrm{C}$.

\subsubsection{Methodische Einschränkungen}

Diese Studie beruht auf Befunden, die im Rahmen von Windkraftplanungen an fünf Standorten in Bergwäldern der Steiermark erhoben wurden. Um einen größeren Datensatz statistisch auswerten zu können, wäre eine Erhebung längerer Zeitreihen an mehr Standorten vonnöten, idealerweise gekoppelt mit einem akustischen Dauermonitoring in Bodenhöhe. Eine weitere methodische Einschränkung betrifft die teilweise geänderten Schwellenwerteinstellungen von -36 auf $-30 \mathrm{~dB}$. Nach ecoObs haben empirische Tests mit Gondeldaten ergeben, dass dadurch ca. 30-40\% weniger Aufnahmen registriert werden. Es ist daher keine direkte Vergleichbarkeit der Standorte gegeben. Unsicherheit besteht zudem darin, wie die für diese Höhenlage hohen Nachttemperaturen an Standort 5 einzuordnen sind. Der Sommer 2018 war ein Hitzesommer. Trotzdem sind die Werte für Bergwälder ungewöhnlich hoch. Eventuell handelt es sich um einen systematischen Messfehler (z. B. infolge der Nahlage des Sensors an der Gondelabwärme) innerhalb des SCADA-Systems der WEA.

\subsubsection{Konsequenzen für den Windkraftausbau in Bergwäldern}

Die Ergebnisse der vorliegenden Untersuchung zeigen, dass sich die Fledermausaktivität und damit das Kollisionsrisiko an WEA in Bergwaldgebieten von WEA im Tiefland unterscheiden kann. Die Einteilung des Kollisionsrisikos für die Arten bei Hurst et al. $(2015,2016)$ bezieht sich auf Anlagen mit einem Rotorabstand von mehr als $50 \mathrm{~m}$ von der Waldoberkante. Bei geringeren Abständen zur Waldoberkante können auch sonst ungefährdete Arten kollidieren (Hurst et al. 2016; Runkel 2017; Runkel et al. 2018). In den Bergwaldgebieten der Steiermark kommen zu einem Großteil WEA mit niedrigen Rotor-Boden-Abständen zum Einsatz. Zur Überprüfung des festgelegten Abschaltalgorithmus wären daher zusätzlich fallweise Schlagopfersuchen durchzuführen. Aufgrund des schwierigen Geländes ist 
dafür der Einsatz von Suchhunden zu empfehlen. Die Schlagopfersuchen müssen jedenfalls bereits den Monat Juni abdecken, um abzuklären, ob die teils erhöhte Aktivität in diesem Monat auch mit höheren Kollisionsraten korreliert.

Vorläufig sehen wir eine Anwendung der RENEBAT-Daten und damit des ProBat-Tools an Bergwaldstandorten kritisch. Die Ergebnisse dieser Untersuchung zeigen, dass die Aktivitätsmuster in Bergwäldern teilweise von dem Forschungsvorhaben RENEBAT abweichen. So bestehen Unterschiede in der Artenzusammensetzung; der Anteil der Gruppe Nyctaloid liegt um einiges höher als in den RENEBAT-Studien. Ein weiterer Unterschied betrifft die Aktivität im Jahresverlauf. Erhöhte Aktivitäten treten an Bergwaldstandorten ab Juni auf, während die Algorithmen von RENEBAT hohe Abschaltzeiten vor allem für die Spätsommer- und Herbstmonate Juli bis September vorsehen (Behr et al. 2011). Es wäre daher notwendig, ein eigenes Modell zur Aktivitätsvorhersage an Bergwaldstandorten zu entwickeln.

Gängige Richtwerte für Abschaltalgorithmen sollten jedenfalls nicht uneingeschränkt vom Tiefland auf Bergwaldgebiete übertragen werden. Die hohe Variabilität der Fledermausaktivität spricht gegen einheitliche Faustregeln und für eine spezifische Bewertung jedes einzelnen Standorts. Zusätzliche Untersuchungen wären - wie oben angeregt - daher wünschenswert, um die Evidenzbasis zu systematischen Unterschieden zwischen Bergwald-, Tiefland- und anderen Gebieten zu erweitern.

Danksagung Für wertvolle Hinweise und die Hilfe bei der Manuskripterstellung danken wir Sebastian Seebauer sowie den beiden Gutachtern, für die Unterstützung bei den Bestimmungsarbeiten Daniela Wieser, für die Korrektur der englischen Zusammenfassung Dominik Heinrici. Besonderer Dank gilt den Anlagenbetreibern von vier Standorten für die Zustimmung zur Verwendung der Witterungsdaten für diesen Beitrag. Aus Gründen der Vertraulichkeit werden hier weder die Anlagenbetreiber noch die Anlagenstandorte genannt.

Die Veröffentlichung wurde durch den Open-Access-Publikationsfonds für Monografien der Leibniz-Gemeinschaft gefördert.

\section{Literatur}

AG Fledermäuse und Windenergie (2014) Positionspapier Fledermäuse und Windenergie, Koordinationsstelle für Fledermausschutz und -forschung in Österreich (KFFÖ)

Ahlen I, Baggøe HJ (1999) Use of ultrasound detectors for bat studies in Europe: experiences from field identification, surveys, and monitoring. Acta Chiropterol 1:137-150

Amt der Steiermärkischen Landesregierung (2017) Klima- und Energiestrategie Steiermark 2030, Graz

Arnett EB, Brown WK, Erickson WP, Fiedler JK, Hamilton BL, Henry TH, Jain A, Johnson GD, Kerns J, Koford RR, Nicholson CP, O’Connell TJ, Piorkowski MD, Tankersley RD Jr, (2008) Patterns of Bat Fatalities at Wind Energy Facilities in North America. J Wildl Manag 72:61-78

Behr O, Brinkmann R, Niermann I, Korner-Nievergelt F (2011) Akustische Erfassung der Fledermausaktivität an Windenergieanlagen. In: Brinkmann R, Behr O, Niermann I, Reich M (Hrsg) Entwicklung von Methoden zur Untersuchung und Reduktion des Kollisionsrisikos 
von Fledermäusen an Onshore-Windenergieanlagen. Umwelt und Raum, Bd 4. Cuvillier, Göttingen

Behr O, Brinkmann R, Korner-Nievergelt F, Nagy M, Niermann I, Reich M, Simon R (Hrsg) (2015) Reduktion des Kollisionsrisikos von Fledermäusen an Onshore-Windenergieanlagen (RENEBAT II). Umwelt und Raum, Bd 7. Institut für Umweltplanung, Hannover

Behr O, Brinkmann R, Hochradel K, Mages J, Korner-Nievergelt F, Reinhard H, Simon R, Stiller F, Weber N, Nagy M (2018) Bestimmung des Kollisionsrisikos von Fledermäusen an Onshore-Windenergieanlagen in der Planungspraxis (RENEBAT III). Universität Erlangen-Nürnberg, Endbericht

BMNT, BMVIT (2018) \#mission2030 Die österreichische Klima- und Energiestrategie. Bundesministerium Nachhaltigkeit und Tourismus und Bundesministerium Verkehr, Innovation und Technologie, Wien

Bontadina F, Beck A, Dietrich A, Dobner M, Eicher C, Frey-Ehrenbold A, Krainer K, Loercher F, Maerki K, Mattei-Roesli M, Mixanig H, Plank M, Vorauer A, Wegleitner S, Widerin K, Wieser D, Wimmer B, Reiter G (2014) Massive bat migration across the alps: implications for wind energy development. In: Abstracts of the 13th European Bat Research Symposium, Sibenik, 1-5 September 2014: 41

Brinkmann R, Behr O, Niermann I, Reich M (Hrsg) (2011) Entwicklung von Methoden zur Untersuchung und Reduktion des Kollisionsrisikos von Fledermäusen an Onshore-Windenergieanlagen. Umwelt und Raum, Bd 4. Cuvillier, Göttingen

Dietz C, Nill D, Helversen O v. (2016) Handbuch der Fledermäuse Europa und Nordwestafrika. Kosmos, Stuttgart

Dürr T (2019) Fledermausverluste an Windenergieanlagen/bat fatalities at wind turbines in Europe. Daten aus der zentralen Fundkartei der Staatlichen Vogelschutzwarte im Landesamt für Umwelt Brandenburg, Stand 07. Januar 2019. https://lfu.brandenburg.de/cms/detail.php/ bb1.c.312579.de

Hurst J, Balzer S, Biedermann M, Dietz C, Dietz M, Höhne E, Karst I, Petermann R, Schorcht W, Steck C, Brinkmann R (2015) Erfassungsstandards für Fledermäuse bei Windkraftprojekten in Wäldern. Diskussion aktueller Empfehlungen der Bundesländer. Natur und Landschaft 90:157-168

Hurst J, Biedermann M, Dietz C, Dietz M, Karst I, Krannich E, Petermann R, Schorcht W, Brinkmann R (Hrsg) (2016) Fledermäuse und Windkraft im Wald: Ergebnisse des F+E Vorhabens (FKZ 351284 0201) „Untersuchungen zur Minderung der Auswirkungen von WKA auf Fledermäuse, insbesondere im Wald“. Naturschutz Biologische Vielfalt 153: 1-396

Kugelschafter K (2013) Windenergie: „Schlagende Argumente“ für den Artenschutz. Positionspapier von Fledermaus-Experten. Naturschutz Landschaftsplanung 45:62-63

Lehnert LS, Kramer-Schadt S, Schönborn S, Lindecke O, Niermann I, Voigt CC (2014) Wind farm facilities in Germany kill Noctule Bats from Near and Far. PLoSONE 9:e103106. https://doi.org/10.1371/journal.phone.0103106

Lindemann C, Runkel V, Kiefer A, Lukas A, Veith M (2018) Abschaltalgorithmen für Fledermäuse an Windenergieanlagen. Eine naturschutzfachliche Bewertung. Naturschutz Landschaftsplanung 50:418-425

Pfalzer G (2017) Der Kleine Abendsegler (Nyctalus leisleri KUHL, 1817) in der Pfalz - ein Opfer der Energiewende? (Mammalia: Chiroptera). Fauna Flora in Rheinland-Pfalz 13:761-777

Presetnik P, Šalamun A (2019) First records of the European free-tailed bat Tadarida teniotis (Rafinesque, 1814) in Slovenia. Natura Sloveniae 21:47-53

Reichenbach M, Brinkmann R, Kohnen A, Köppel A, Menke J, Ohlenburg H, Reers H, Steinborn H, Warnke M (2015) Bau- und Betriebsmonitoring von Windenergieanlagen im Wald. Abschlussbericht, Bundesministeriums für Wirtschaft und Energie, Berlin

Runkel V (2017) Minderungsmassnahmen an WEA: Ausreichender Schutz der Fledertiere? https://www.volkerrunkel.de/Poster-Tagung-Echolot-2017.pdf 
Runkel V, Gerding G, Marckmann U (2018) Handbuch: Praxis der akustischen Fledermauserfassung. tredition, Hamburg

Rydell J, Engström H, Hedenström A, Larsen JK, Pettersson J, Green M (2012) The effect of wind power on birds and bats. A synthesis. Report 6511, Swedish Environmental Protection Agency, Stockholm

Santos H, Rodrigues L, Jones G, Rebelo H (2013) Using species distribution modelling to predict bat fatality risk at wind farms. Biol Cons 157:178-186

Voigt CC, Popa-Lisseanu AG, Niermann I, Kramer-Schadt S (2012) The catchment area of wind farms for European bats: a plea for international regulations. Biol Cons 153:80-86

Voigt CC, Lehnert LS, Petersons G, Adorf F, Bach L (2015) Wildlife and renewable energy: German politics cross migratory bats. Eur J Wildl Res. https://doi.org/10.1007/s10344-0150903-y

Widerin K, Jerabek M (2014) Fledermausnachweise am Kalser Törl (2.518 m, Hohe Tauern, Salzburg). Berichte der Naturwissenschaftlich-Medizinischen Vereinigung Salzburg 17:33-42

Widerin K, Reiter G (2017) Bat activity at high altitudes in the Central Alps, Europe. Acta Chiropterol 19:379-387

Widerin K, Reiter G (2018) Bat activity and bat migration at the elevation above 3,000 $\mathrm{m}$ at Hoher Sonnblick massive in the Central Alps, Austria (Chiroptera). Lynx 49:223-242

Zahn A, Lustig A, Hammer M (2014) Potenzielle Auswirkungen von Windenergieanlagen auf Fledermauspopulationen. ANLiegen Natur 36:21-35

Zingg PE, Bontadina F (2016) Migrating bats cross top of Europe. PeerJ Preprints 4:e2557v1. https://doi.org/10.7287/peerj.preprints.2557v1

Open Access Dieses Kapitel wird unter der Creative Commons Namensnennung 4.0 International Lizenz (http://creativecommons.org/licenses/by/4.0/deed.de) veröffentlicht, welche die Nutzung, Vervielfältigung, Bearbeitung, Verbreitung und Wiedergabe in jeglichem Medium und Format erlaubt, sofern Sie den/die ursprünglichen Autor(en) und die Quelle ordnungsgemäß nennen, einen Link zur Creative Commons Lizenz beifügen und angeben, ob Änderungen vorgenommen wurden.

Die in diesem Kapitel enthaltenen Bilder und sonstiges Drittmaterial unterliegen ebenfalls der genannten Creative Commons Lizenz, sofern sich aus der Abbildungslegende nichts anderes ergibt. Sofern das betreffende Material nicht unter der genannten Creative Commons Lizenz steht und die betreffende Handlung nicht nach gesetzlichen Vorschriften erlaubt ist, ist für die oben aufgeführten Weiterverwendungen des Materials die Einwilligung des jeweiligen Rechteinhabers einzuholen.

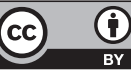

